KLEIST-JAHRBUCH 1992 


\section{KLEIST-JAHRBUCH 1992}

Herausgegeben von

Hans Joachim Kreutzer

VERLAG J. B. METZLER STUTTGART · WEIMAR 
Anschrift des Herausgebers:

Universität Regensburg, Institut für Germanistik

Postfach 101042, D-8400 Regensburg 1

Redaktion: Ernst Osterkamp

Die Deutsche Bibliothek - CIP-Einheitsaufnabme

Kleist-Jahrbuch ... / - Stuttgart: Metzler.

Erscheint jährlich. - Früher im Verl. E. Schmidt, Berlin. -

Aufnahme nach 1990 (1991)

ISSN 0722-8899

1990 (1991) -

Verl.-Wechsel

ISBN 978-3-476-00872-5

ISBN 978-3-476-03444-1 (eBook)

DOI 10.1007/978-3-476-03444-1

Dieses Werk einschließlich aller seiner Teile ist urheberrechtlich geschützt.

Jede Verwertung außerhalb der engen Grenzen des Urheberrechtsgesetzes ist ohne Zustimmung des Verlages unzulässig und strafbar. Das gilt insbesondere für Vervielfältigungen, Übersetzungen, Mikroverfilmungen und die Einspeicherung und Verarbeitung in elektronischen Systemen.

(C) 1992 Springer-Verlag GmbH Deutschland

Ursprünglich erschienen bei J. B. Metzlersche Verlagsbuchhandlung und Carl Ernst Poeschel Verlag GmbH 1992 


\section{INHALT}

Vorwort $\ldots \ldots \ldots \ldots \ldots \ldots \ldots \ldots \ldots \ldots \ldots \ldots \ldots \ldots \ldots \ldots, \quad$ VII

\section{Kleist-Preis 1991}

Dieter GöRne (Dresden): Zur Begrüßung. $\ldots \ldots \ldots \ldots \ldots \ldots \ldots \ldots \ldots$

Hans Joachim Kreutzer (Regensburg): Rede zur Verleihung des Kleist-

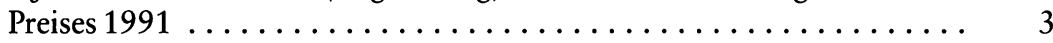

HANS MAgnus EnZENSBerger (München): Rede auf Gaston Salvatore .... 7

Gaston Salvatore (Venedig): Die Verbannten. Der Künstler und sein Land. Rede anläßlich der Entgegennahme des Kleist-Preises . . . . . . . . . .

\section{Abhandlungen}

HANs ZelLeR (Fribourg): Die Berliner Kleist-Ausgabe und die Standards der Editionsphilologie.

HANS-Jürgen SCHRADER (Genf): Ein anderer K.? Die >Beiträge zur KleistForschung $1974-1990$. Ein Resümee ....................

Peter Michelsen (Heidelberg): Die Betrogenen des Rechtgefühls. Zu Kleists

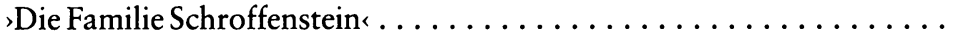

Hans-Georg Werner (Halle): Geschichtlichkeit in Kleists 'Prinz Friedrich von Homburg . . . . . . . . . . . . . . . . . . . . . . . . .

Klaus Peter (Amherst): Für ein anderes Preußen. Romantik und Politik in

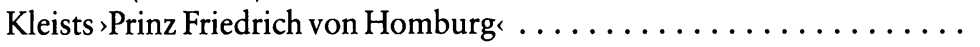

PETER HoRn (Kapstadt): "... sich träumend, seiner eignen Nachwelt gleich.. " Verhinderte Tragik im Traum des Prinzen Friedrich von Homburg von seinem postumen Ruhm. . . . . . . . . . . . . . . . . . . . . 
Raymond Lucas (Oxford): Die Aporie der Macht. Zum Problem der Amnestie in Kleists Michael Kohlhaas . . . . . . . . . . . . . . . . . . . . 140

Wolfgang Wittkowski (Albany): Gerechtigkeit und Loyalität, Ethik und Politik. Kleists > Verlobung in St. Domingo < und Goethes teilweiser Widerspruch in der $>$ Belagerung von Mainz $\ldots \ldots \ldots \ldots \ldots \ldots \ldots \ldots$

KrZYsztof Lipı́́ski (Krakau): Heinrich von Kleist in Polen. . . . . . . . . . . 172

Roger Paulin (Cambridge): Kleist in Großbritannien. Aus Anlaß von Mary Howards Buch $>$ Vom Sonderling zum Klassiker . . . . . . . . . . . . .

HANS JoACHIM KREUTZER (Regensburg): "... der erste nationalsozialistische Dichter der Vergangenheit ..... Georg Minde-Pouets Krisenbericht von 1936..................................

\section{Rezensionen}

Peter Goldammer (Weimar) über Heinrich von Kleist: Sämtliche Werke und Briefe. Bd. 1. Dramen 1802-1807. Unter Mitwirkung von Hans Rudolf Barth hg. von Ilse-Marie Barth und Hinrich C. Seeba. . . . . . . . . . . .

Bernd Leistner (Leipzig) über Wolf Kittler: Die Geburt des Partisanen aus dem Geist der Poesie . . . . . . . . . . . . . . . . . . . . . .

Anschriften der Mitarbeiter . . . . . . . . . . . . . . . . 205 


\section{VORWORT}

Dieser Band des Kleist-Jahrbuchs ist der erste, der ohne thematische Vorgaben zustande gekommen ist. Er dokumentiert keine Veranstaltung, sein Inhalt beruht ausschließlich auf dem freien Angebot der Autoren. Damit entspricht das KleistJahrbuch 1992 als bisher einziger Band dieser Reihe der üblichen Publikationsform von Jahrbüchern, die im allgemeinen nichts anderes darstellen als eine normale Fachzeitschrift, mit dem Unterschied, daß sie als geschlossener Band erscheinen.

Durch den Sonderfall dieses Zwischenjahres sieht sich der Herausgeber in seinen Aufgaben wesentlich erleichtert. Die Rubrik der "Freien Beiträge « muß für gewöhnlich schmal gehalten werden, weil die stets über Jahre hin vorausgeplanten Kolloquien der Heinrich-von-Kleist-Gesellschaft den größten Teil des zur Verfügung stehenden Raumes in Anspruch nehmen. Diese Beschränkung ist ein undankbares Geschäft. Dagegen wurde es jetzt möglich, gleichsam den freien Diskussionsstrom selbst zu einem bestimmten Zeitpunkt zu beobachten. Womit beschäftigen sich Literaturwissenschaftler unter dem Vorzeichen Kleist - sofern sie nicht von der Heinrich-von-Kleist-Gesellschaft um Beiträge geradezu gebeten werden? Antwort: sie debattieren ausgiebig miteinander und übereinander und gelegentlich streitbar. Das ist keine Diskussion um Tagesthemen, wie sie Zeitungen ansteht, sondern es zeigt sich, daß auch scheinbar Vergessenes durchaus lebendig ist. Die Tatsache etwa, daß die Theater keinen Zugang zu Kleists kompliziertester Tragödie, der 'Familie Schroffenstein', finden, hat Peter Michelsen nicht gehindert, an die ungelösten Fragen, die diese gewaltige dramatische Konzeption aufwirft, zu erinnern. - Daß man in Frankfurt an der Oder seit zwei Jahrzehnten in den Grenzen der politischen Vorgaben nach Kräften auch mit Publikationen für Kleist gestritten hat, wurde kontinuierlich beobachtet, Hans-Jürgen Schrader hat das alles jetzt sorgfältig und systematisch analysiert und stellt es hier erstmals vor. - Andere Grenzen waren stets leichter zu überschreiten gewesen. Über Kleist in Polen hatte Olga Dobijanka schon 1981 in Berlin berichtet. Jetzt liefert ihr Schüler Krzysztof Lipiński, gleichfalls an der Jagiellonischen Universität in Krakau tätig, aber in der Zwischenzeit als Humboldt-Stipendiat auch Gast der Universität Regensburg, die Fortsetzung dazu: Zeichen einer gewachsenen fachlichen und nicht zuletzt freundschaftlichen Verbindung. - In ähnlichem Sinne würdigt Roger Paulin (Cambridge) die Geschichte der Wirkung Kleists in England, wie sie in einer von Klaus Kanzog betreuten Dissertation nachgezeichnet wurde.

Die ungewöhnliche Aktualität der Abhandlung Hans Zellers über die bisherige Leistung der "Berliner Kleist-Ausgabe « ist ihr zum Teil aus der Zeitungsdiskussion über ihre Finanzierungsprobleme zugewachsen, insoweit ist die Aktualität zufällig. 
Die hier erörterten Fachfragen hingegen sind alles andere als Tagesfragen. Das KleistJahrbuch hat sich dieser Edition gegenüber bisher Zurückhaltung auferlegt. Eine einzige Rezension (von Klaus Kanzog, Kleist-Jahrbuch 1990, zu den Bänden II,2 und II,4) ist erschienen. Der Herausgeber ist zur Neutralität verpflichtet, denn das KleistJahrbuch wird im Auftrage des Vorstandes der Heinrich-von-Kleist-Gesellschaft herausgegeben, die Kleist-Gesellschaft selbst aber ist kein Diskutant in wissenschaftlichen Fragen, ebensowenig wie andere Institutionen. Mit Hans Zeller hat ein Unabhängiger sein Urteil abgegeben: er ist auf dem Felde der Editionsphilologie seit langem der mutmaßlich angesehenste Fachmann, er ist Ausländer, gehört der Heinrich-von-Kleist-Gesellschaft nicht an und ist überdies Präsident des Kuratoriums der Frankfurter Hölderlin-Ausgabe, die im selben Verlag erscheint wie die »Berliner Kleist-Ausgabe «. Das Resultat lautet, in einem Satz zusammengefaßt: die Beurteilungen der Wissenschaftler und die der Zeitungsschreiber fallen grundsätzlich auseinander, zwischen beiden besteht keinerlei argumentative Brücke. Aus fachlichen Gesichtspunkten auf der einen Seite und politischen Tendenzen auf der andern kann sich kein Diskurs bilden.

Die Dokumentation der Verleihung des Kleist-Preises 1991 an Gaston Salvatore in Dresden, die diesen Band eröffnet, vermag das einzigartige und unwiederholbare Echo der Veranstaltung im Schauspielhaus nicht festzuhalten. Die Wirkung der Reden Enzensbergers und Salvatores in einem Lande, das schlechterdings keine öffentliche Rede kannte, ausgenommen in der Sprache der Partei-und der der Kunst, entzieht sich der Beschreibung. Hoyerswerda, wo damals Ausländer mißhandelt worden waren, liegt nicht weit von Dresden entfernt. An Ort und Stelle hat man die Geste Enzensbergers, der den Fremden gleichsam in die deutsche Literatur einband, vollkommen verstanden.

Ein düsterer Blick in die Vergangenheit eröffnet sich dem, der heute noch an den Schutt und Staub des Dritten Reiches rührt. Bewußtmachen ist aber mit Sicherheit der erste Schritt in die Zukunft. Mag es auch inzwischen um die Generation unserer Großväter gehen: niemand vermag uns das abzunehmen. Georg Minde-Pouet hat im Dritten Reich die Stimme der früheren Kleist-Gesellschaft nachdrücklich artikuliert, als er 1933 den Vorsitzenden dieser Gesellschaft, Oskar Walzel, aus dem Amt brachte. Walzel, mit einer Jüdin verheiratet, hat in Bonn mit Mühe noch einige Jahre sein Leben gefristet.

Der Herausgeber darf konstatieren, daß die heimliche Kennmarke des KleistJahrbuchs, das Mitarbeiterverzeichnis, bei freier Beiträgerschaft nicht anders aussieht als bei Texten, die auf Anstöße der Kleist-Gesellschaft zurückgehen. Von Kapstadt bis Massachusetts und von Krakau bis Genf ist das Netz gespannt, ja der Kenner der akademischen Welt wird mit Aufmerksamkeit notieren, daß Cambridge und Oxford darin vorkommen ... 